\title{
Lossless Compression of Pharynx and Esophagus in Fluoroscopic Medical Images
}

\author{
Arif Sameh Arif, Sarina Mansor, Rajasvaran Logeswaran, and Hezerul Abdul Karim
}

\begin{abstract}
Hospitals and medical centers produce a tremendous amount of sequential images for medical examinations such as MRI, CT and Fluoroscopy. This series of images takes up a large amount of storage space, in addition to the cost and time incurred during transmission. For medical data, lossless compression is preferable to the greater gains of lossy compression, in the interest of reliability. This paper proposes a new method for lossless compression of pharynx and esophagus fluoroscopy images, depending on correlation and combination of Run Length and Huffman. Otherwise, the shifted images moved to a shifted group and compress separately. From the experimental results obtained, the proposed method achieved improved performance with a compression ratio of $\mathbf{1 2 . 2}$ for the proposed combination of Run-length and Huffman coding (R. Huff) on the difference images as compared to 1.35 for the standard method.
\end{abstract}

Index Terms-Fluoroscopy, ROI, lossless image compression, run-length coding, huffman coding, correlation.

\section{INTRODUCTION}

In the last decade medical images have been tremendously increased in terms of generation, transmission and storage. This has brought the obsession of many researchers in developing approaches for compressing medical images.

Compression techniques can be split into two main categories: lossy and lossless. Lossy compression reduces the accuracy of medical images, and doctors need accurate information to diagnose the case of the patient. For that reason, researchers have concentrated on lossless compression for medical applications [1].

Medical images can be classified into two main models: single and sequential images. One of the famous types of single image is X-ray, while sequential images include Computed Tomography (CT), Magnetic Resonance Imaging (MRI) and Fluoroscopy. By applying different categories of compression methods, it has seen found that good results could be gained in lossless compression [2].

Fluoroscopy is a special type of X-ray that provides continuous X-ray images of a patient's organ structures in real-time. It is used in many types of examinations and procedures, such as cardiac catheterization, Percutaneous Nephrostomy (PCN), Barium Swallow, and others [3].

The correlation coefficient (CC) is one of the most common similarity measures, and it helps highlight changes. CC measures the dependences existing between two quantities, and it has been used to express the quality of a least squares fitting of the sequence of images [4].

Manuscript received March 2, 2013; revised May 3, 2013.

The authors are with Faculty of Engineering, Multimedia University, $63100 \quad$ Cyberjaya, Selangor, Malaysia (email sarina.mansor@mmu.edu.my).
The longstanding popular methods for lossless compression include Huffman and Run Length Encoding (RLE). Huffman coding is a variable length coding that assigns longer codes to symbols with low probabilities and short bit code to those symbols with higher possibilities. This coding scheme is efficient to compress differential data [5]. The RLE is one of the most popular and simplest methods that applied to code the repeated data or code pattern in a single code [6].

The combination of the two effective compression methods RLE and Huffman was proposed to reduce the data volume, pattern delivery time, and save power in scan applications [7]. In medical images, the combination of Run Length and Huffman Coding was implemented on MRI images and X-ray angiograms, to achieve maximum compression [8]. The same combination of Run Length and Huffman coding was implemented for color images after quantization and threshoding the DWT coefficients good results were obtained in [9].

In our recent work, we have performed lossless compression of Fluoroscopy medical images using correlation and Huffman coding (HM-D) [10]. The proposed method achieved compression ratio of 7.97. We extend the work to include the combination of Huffman and Run-length coding. Specifically, we propose a new framework for lossless medical image compression based on classifying the images depending on correlation and coding the difference between sequential images using the combination of Run Length and Huffman coding. The extended method achieved compression ratio of 11.41 [11]. In this paper, we expand the work to enhance the compression ratio by testing the two sequential images to examine the shifting existence. This is to ensure that our proposed method is robust to the shifting cases. To the best of our knowledge, there are no other published works employing this technique in the context of medical image compression.

\section{EXPERIMENTAL WORK}

Identifying and extracting accurately the ROI is an essential step before coding and compressing the image data for efficient transition or storage. By using different spatial regions and identifying the region of interest of the image, it is possible to compress it into different levels of reconstruction quality. Images can be classified into three regions: (1) Primary region of interest (PROI), (2) Secondary region of interest (SROI) and (3) background [12]. This way, one could accurately maintain the features needed for medical diagnosis or scientific measurement, while achieving high overall compression by allowing degeneration of data in the unimportant regions [13]. 
The correlation was determined by the variance and co-variance, where the variance is measured for a dimension with itself, while the covariance is measured between two dimensions. The formula of variance (Var) and co-variance (Cov) are given as follows [14]:

$$
\begin{gathered}
\operatorname{Var}(X)=\frac{\sum_{i}^{n}\left(X_{i}-\bar{X}\right)}{n-1} \\
\operatorname{Cov}(X, Y)=\frac{\sum_{i}^{n}\left(X_{i}-\bar{X}\right)\left(Y_{i}-\bar{Y}\right)}{n-1} \\
R_{X Y}=\frac{\operatorname{Cov}(X, Y)}{\operatorname{Var}(X) \operatorname{Var}(Y)}
\end{gathered}
$$

where $\bar{X}$ and $\bar{Y}$ are the means of $X$ and $Y$ respectively, and $\mathrm{R}$ is the correlation between $X$ and $Y$. To calculate the similarity between the orginal image and the reconstructed image, the correlation coefficient $(C C)$ is calculated as follows:

$$
\begin{aligned}
& C C= \\
& \frac{\sum_{s} \sum_{t}[f(s, t)-\bar{f}(s, t)][w(x+s, y+t)-\bar{w}]}{\left\{\sum_{s} \sum_{t}[f(s, t)-\bar{f}(s, t)]^{2} \sum_{s} \sum_{t}[w(x+s, y+t)-\bar{w}]^{2}\right\}^{1 / 2}}
\end{aligned}
$$

where $x=0,1,2, \ldots, M-1, y=0,1,2, \ldots, N-1, \bar{w}$ is the average value of the pixels in $w, \bar{f}$ is the average value of $f$ (intensity function) in the region coincident with the current location of $w$, and the summations are taken over the coordinates common to both $f$ and $w$, and $M \times \mathrm{N}$ is the size of the original image. The $\mathrm{CC}$ is scaled in the range (-1 to 1$)$, independent of scale changes in the amplitude of $f$ and $w$ [15].

\section{PROPOSED METHOD}

The proposed method is divided into three main phases: the first is classification, the second is preprocessing and the final phase is encoding. To restore the series of images, the process is reversed.

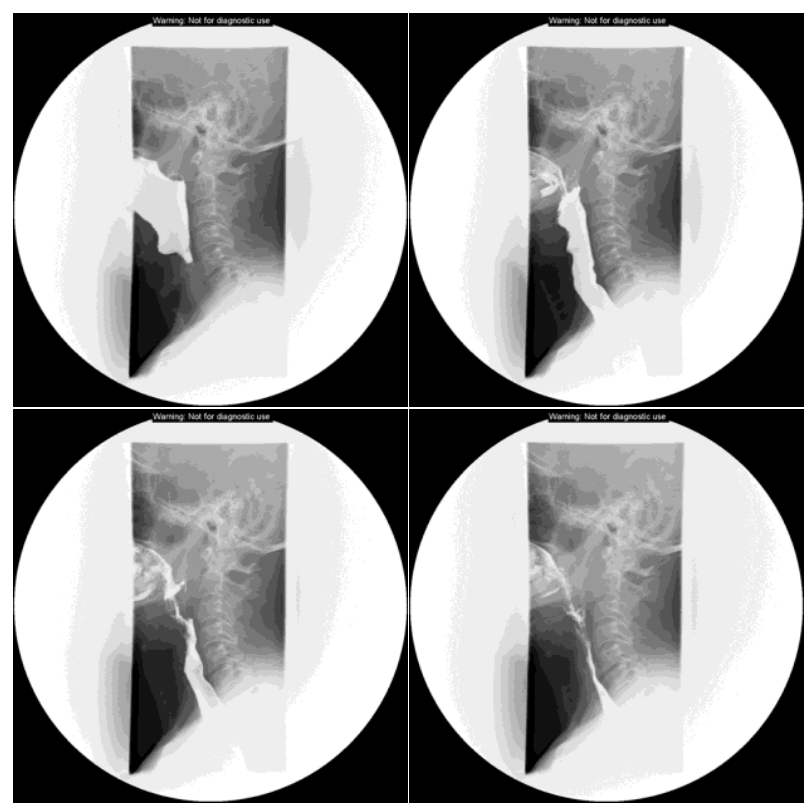

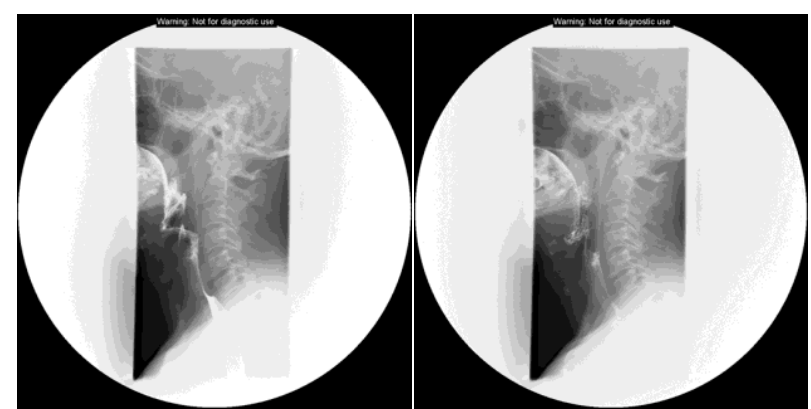

Fig. 1. Set of pharynx and esophagus fluoroscopy images taken from the same angle.

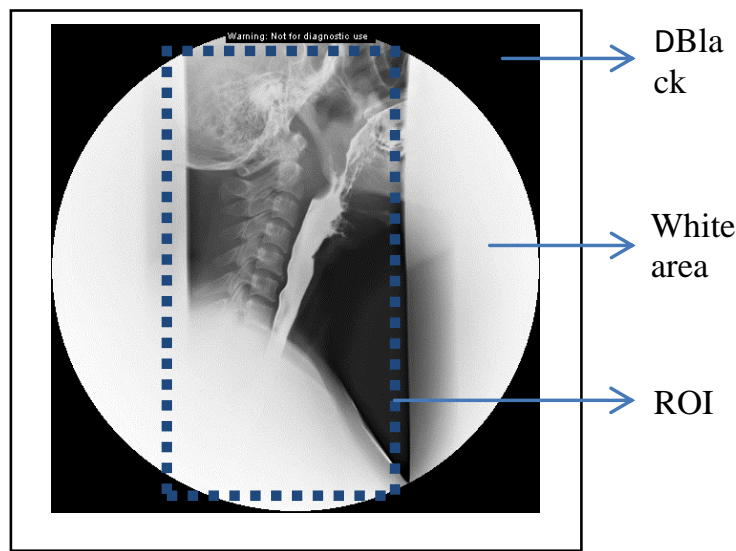

Fig. 2. Important areas in fluoroscopy images.

\section{A. Classification}

Depending on the morphology of the Fluoroscopy images, we need to classify the images of each patient into groups depending on the angle of capture. So, the first step in classification is to generate a group, keeping the first image as a reference image and comparing the other images with it by calculating the CC. This step produces up to three groups for each patient. Duplicated images, including the ones in which the patient moves a little bit, and the radiographer moves, are moved to a shifted group. The vital part in esophagus fluoroscopy images is following the liquid inside the body of the patient. Fig. 3 summarizes the process.

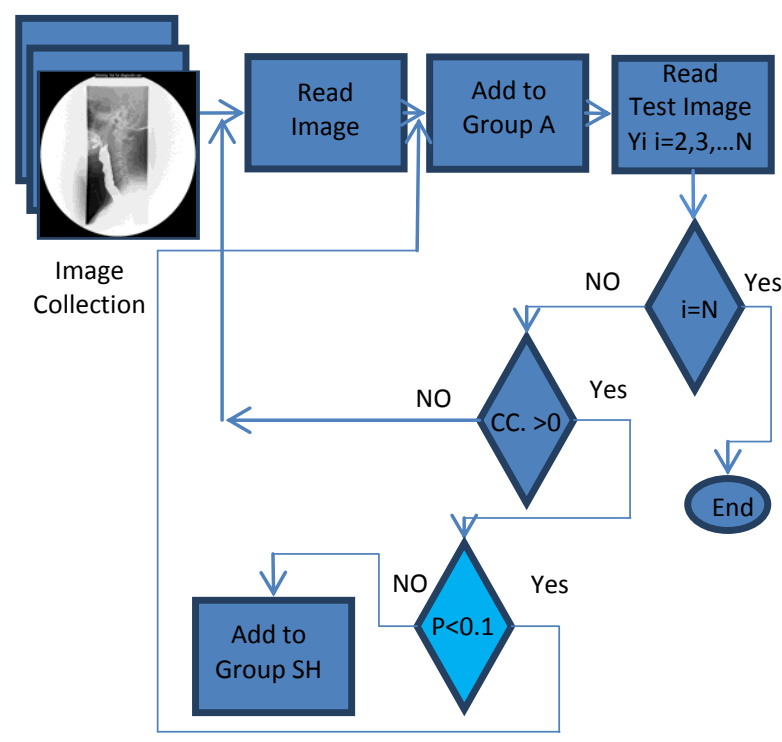

Fig. 3. Classification phase.

\section{B. Preprocessing}

The preprocessing phase can be summarized as follows: 


\section{1) B-1 remove the black areas}

Depending on the aspect of the fluoroscopy images, there are four black corners in each image. By locating the center of the image and calculating the distance from the center to the edge of the image, we can draw a circle that contains the actual data from the fluoroscopy device.

\section{2) B-2 remove the white area}

Depending on the aspect of the fluoroscopy images, there are two white areas in each image. This does not contain any relevant data. Steps B1 - B2 result in the extraction of ROI.

\section{3) B-3 correlation coefficients (CC)}

The next step in this phase is calculating the CC. It is an indicator for the compression ratio means where a high $\mathrm{CC}$ produces high compression ratio.

\section{4) B-4 compute the difference}

Compute the difference between images by subtracting the test image from the reference image, as most images taken from the same view are mostly similar. Therefore, we can use the first image as the base pattern (reference image) and store only the difference results as a vector (see Fig. 4 a) ).

Depending on the circumstance of the shifted group, the steps B3 and B4 cannot be applied on this group because it contain a shifted images and those images do not have any relation between them. Implementing the steps B1 and B2 to extract the ROI and store the images in a vector (see Fig. 4 b) ).
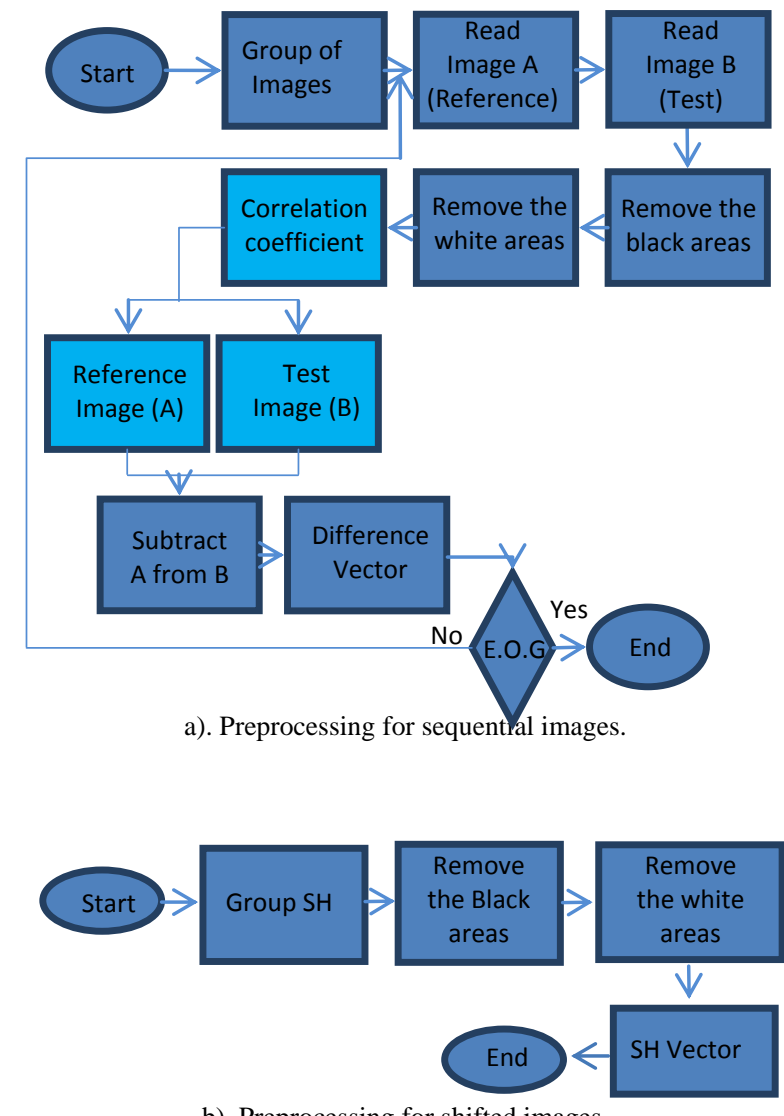

b). Preprocessing for shifted images. Fig. 4. Preprocessing phase.

\section{Encoding}

The preprocessing (phase B) produces a one dimensional vector. Depending on the structure of the vector data, we can decide the method of coding. The combination of Run Length and Huffman coding is selected because they are based on repetition and the estimated probability of occurrence for each possible value of the source symbol (see Fig. 5 a) ).

Depending on the characteristic of the $\mathrm{SH}$ vector the combination of R. Huff did not give a good resutls, for this reson applying Huff encoding gaine a good results( see Fig. 5 b) ).

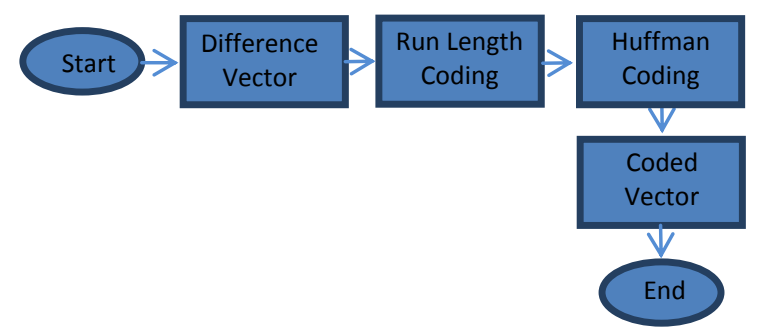

a). Encoding the sequential images.

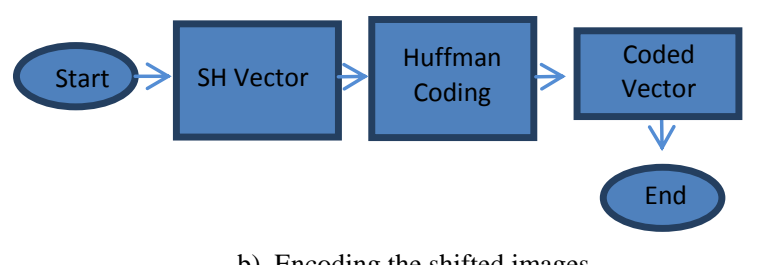

b). Encoding the shifted images. Fig. 5. Encoding phase.

\section{RESUlTS AND DISCUSSION}

Experiments were performed on 114 fluoroscopy images, each of size $512 \times 512$ pixels and file size of $256 \mathrm{~KB}$, to evaluate the validity of the proposed approach. The performance evaluation, in terms of Compression Ratio $(C R)$ depending on the $\mathrm{CC}$, is tabulated in Table 1 . The $\mathrm{CC}$ value is calculated as in (4), while the $C R$ is calculated as follows:

$$
C R=\frac{S_{o}}{S_{d}}
$$

where

$S_{o}=$ File size of the original image,

$S_{d}=$ File size of the difference image.

Table I shows the correlation values between the tested and the reference images for a random sample of twenty six images. The higher correlation is correlated with higher redundancy that will give higher compression ratio.

From Table I, it is observed that the proposed method ( $\mathrm{R}$. Huff-P) achieved significantly better performance than implementing Huffman coding on the standard images (HM-S), Huffman coding on the difference images (HM-D) and it also performed better than the standard lossless combination (R. Huff-S) compression of the images. For example, images 30 and 31 , the standard combination method (R. Huff-S) only produced CR of 1.35. Huffman coding on the standard images (HM-S) gained a CR of 1.15, Huffman coding on the difference images (HM-D) gained a CR of 6.92 but the proposed method (R. Huff-P) implemented based on the CC indications, a CR of 12.2 was achieved for the difference image (30-31). Assuming that image 30 was stored in the standard method, the improvement in CR for storing the vital information of image 31 losslessly improved from 1.35 to 12.2 , which is significant 900\% improvement. 
TABle I: COMPARISON OF COMPRESSION Ratio PeRFORMANCE (CR) BETWEen tHE PROPOSED METHOD THE COMBINATION OF RUN-LeNGTH AND HuFFMAN CODING (R. HuFf-P) ON THE DifFERENCE IMAGES AS COMPARED TO THE IMPLEMENTATION OF HUFFMAN CODING ON THE Standard Images (HM-S), HuffMan Coding on the Difference IMAGES (HM-D) AND TO THE COMBINATION OF RUN LENGTH AND HUFFMAN CODING (R. HUFF-S) ON THE STANDARD IMAGES FOR A RANDOM SAMPLE OF TEN IMAGES. [CC IS THE CORRELATION BETWEen THE TESTED (TEST) AND THE REFERENCE (REF.) IMAGES]

\begin{tabular}{ccccccc}
\hline & & & \multicolumn{4}{c}{ Compression Ratio (CR) } \\
\cline { 5 - 7 } Ref. & Test & CC & HM-S & $\begin{array}{c}\text { R. } \\
\text { Huff-S }\end{array}$ & HM-D & $\begin{array}{c}\text { R. } \\
\text { Huff-p }\end{array}$ \\
\hline 3 & 4 & 0.96 & 1.13 & 1.35 & 5.36 & 4.70 \\
\hline 9 & 10 & 0.96 & 1.15 & 1.31 & 5.6 & 6.09 \\
\hline 8 & 9 & 0.95 & 1.17 & 1.36 & 4.42 & 2.52 \\
\hline 19 & 20 & 0.95 & 1.12 & 1.37 & 5.92 & 7.41 \\
\hline 21 & 22 & 0.94 & 1.16 & 1.34 & 6.53 & 7.15 \\
\hline 7 & 8 & 0.93 & 1.16 & 1.36 & 6.3 & 9.56 \\
\hline 10 & 11 & 0.92 & 1.14 & 1.35 & 5.49 & 5.59 \\
\hline 6 & 7 & 0.91 & 1.15 & 1.35 & 6.44 & 10.12 \\
\hline 30 & 31 & 0.91 & 1.15 & 1.35 & 6.92 & 12.2 \\
\hline 25 & 26 & 0.90 & 1.16 & 1.35 & 5.87 & 6.78 \\
\hline 12 & 13 & 0.90 & 1.13 & 1.31 & 5.85 & 6.86 \\
\hline 29 & 30 & 0.90 & 1.15 & 1.35 & 5.16 & 4.71 \\
\hline 27 & 28 & 0.89 & 1.15 & 1.35 & 6 & 7.99 \\
\hline 4 & 5 & 0.89 & 1.12 & 1.35 & 3.92 & 2.28 \\
\hline 18 & 19 & 0.89 & 1.12 & 1.36 & 5.88 & 8.01 \\
\hline 22 & 23 & 0.89 & 1.16 & 1.35 & 5.39 & 6.59 \\
\hline 28 & 29 & 0.89 & 1.15 & 1.31 & 5.92 & 7.08 \\
\hline 14 & 15 & 0.89 & 1.11 & 1.33 & 5.79 & 6.03 \\
\hline 26 & 27 & 0.89 & 1.16 & 1.32 & 5.14 & 4.69 \\
\hline 5 & 6 & 0.88 & 1.18 & 1.36 & 5.85 & 7.74 \\
\hline 24 & 25 & 0.87 & 1.16 & 1.34 & 5.83 & 7.59 \\
\hline 15 & 16 & 0.85 & 1.12 & 1.31 & 4.21 & 2.84 \\
\hline 23 & 24 & 0.82 & 1.16 & 1.35 & 4.92 & 4.71 \\
\hline 16 & 17 & 0.77 & 1.13 & 1.34 & 3.84 & 2.57 \\
\hline 17 & 18 & 0.70 & 1.13 & 1.32 & 1.8 & 2.15 \\
\hline 13 & 14 & 0.67 & 1.14 & 1.31 & 4.33 & 3.52 \\
\hline \hline & & & & & & \\
\hline
\end{tabular}

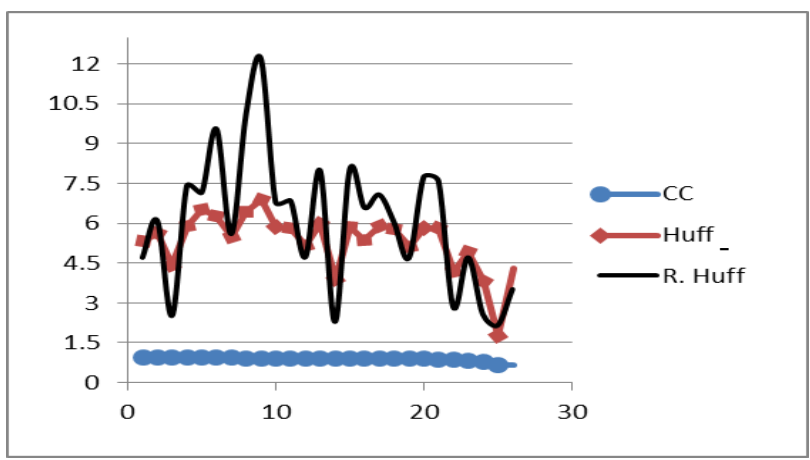

a). Before applying the proposed method on the shifted images.

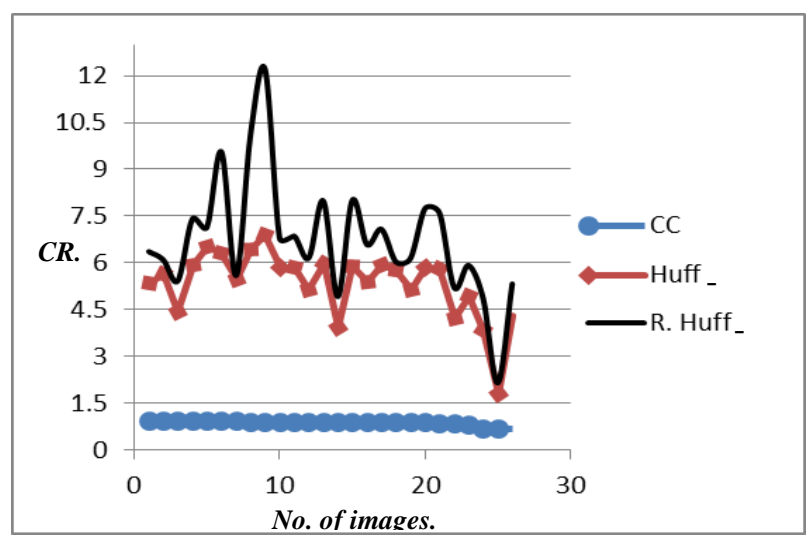

b). After applying the proposed method on the shifted images.

Fig. 6. Comparative analysis between Huffman and a combination of Run Length Huffman depending on Correlation before coding the shifted images in the proposed method and after applying the proposed method onto the shifted images.
As a comparison in Fig. 6 between a, and b, it is obviously seen that the performance of the R. Huff in some images comes lower than the performance of Huff alone. This reduction in the performance may be caused by the duplicated images that the radiographer may recapture the same image of the same liquid movement, because captured one may has a little bit shifting. This shifting may be caused by a slice motion that may come from the patient or the radiographer himself. To solve this problem, shifted images were isolated in a group to be compressed using Huffman coding only. Preliminary results showed that these images got a compression ratio using Huffman only higher than the same ratio used proposed method. The main idea of the proposed method is the subtraction between two similar images that have a lot equal corresponding pixels and some areas of moving liquid. This similarity results a lot of non-sequential values; Run length utilizes these sequential values. In shifted images, although they have similar view, the corresponding pixels are not equal, which means a lot of non-zero values; in this case, Run Length does not give a high compression ratio, which may affect the total compression ratio of the proposed method. In which Huffman alone gives higher compression ratio than $\mathrm{R}$. Huff.

\section{CONCLUSION}

In this paper, a new framework for image compression based on grouping the images reliant on the correlation has been proposed. The technique concentrates on the region of interest to code the difference between the groups of images using the combination of Run-length and Huffman coding. The method has achieved significant improvement in compression performance, and indirectly storage and transmission benefits. Our proposed framework is not coding specific, we would like to implement other lossless coding techniques such as Golomb-rice coding in order to further improve the compression performance.

\section{ACKNOWLEDGEMENT}

The authors would like to thank Dr. Noraini Abdul Rahim (Head Radiology Department), Khatijah Ali (Radiographer) and $\mathrm{Mr}$ Ang Kim Liong (Clinical Research Centre) at Serdang Hospital.

\section{REFERENCES}

[1] S. Bhavani and K. Thanushkodi, "A survey on coding algorithms in medical image compression," International Journal on Computer Science and Engineering, vol. 2, no. 5, pp. 1429-1434, 2010.

[2] M. F. Ukrit, A. Umamageswari, and G. R. Suresh, "A survey on lossless compression for medical images," International Journal of Computer Applications, vol. 31, no. 8, pp. 47-50, Oct. 2011.

[3] C. Mei-Yen, L. Chien-Tsai, S. Ying-Chou, C. Lee Theun, C. Chian-Fa, M. Huo, and C. Cheng-Yen, "Design and evaluation of a DICOM compliant video fluoroscopy imaging system," in Proc. IEEE 9th International Conference on Health Networking Application and Services, Taipei, June 2007, pp. 248-251.

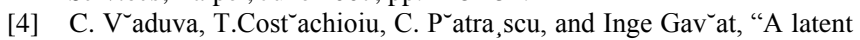
analysis of earth surface dynamic evolution using change map time series," in Proc. IEEE Transaction on Geoscience and Remote Sensing, vol. 51, no. 4, April 2013, pp. 2105-2118.

[5] T. Song and T. Shimamoto, "Reference frame data compression method for H.264/AVC," IEICE Electronics Express, vol. 4, no. 3, pp. 121-126, 2007.

[6] J. L. $\mathrm{Nu}^{\prime} \mathrm{n}^{\sim}$ ez and S. Jones, "Run-length coding extensions for high performance hardware data compression," IEE Proceeding-Computer Digital Techniques, vol. 150, no. 6, 2003, pp. 387-395. 
[7] M. Norani and M. H. Tehranipour, "RL-Huffman encoding for test compression and power reduction in scan applications," ACM. Trans. USA, vol. 10, issue 1, pp. 91-115, 2005.

[8] R. S. Sunder, C. Eswaran, and N. Sriraam, "Performance evaluation of 3-D transforms for medical image compression," in Proc. IEEE International Conference, Electro Information Technology, Lincoln, NE, vol. 6, May 2005, pp. 6.

[9] V. Setia and V. Kumar, "Coding of DWT coefficients using Run-length coding and Huffman coding for the purpose of color image compression," International Journal of Computer and Communication Engineering, vol. 6, pp. 201-204, 2012.

[10] A. S. Arif, S. Mansor, R. Logeswaran, and H. A. Karim, "Lossless compression of fluoroscopy medical images using correlation," Journal of Asian Scientific Research, vol. 11, no. 2, pp. 718-723, 2012.

[11] A. S. Arif, S. Mansor, R. Logeswaran, and H. Abdul Karim, "Lossless compression of fluoroscopy medical images using correlation and the combination of Run Length and Huffman coding," in Proc. IEEE International Conference on Biomedical Engineering \& Science IECBES, December 2012.

[12] P. Akhtar, M. I. Bhatti, T. Javid Ali, and M. Abdul Muqeet, "Significance of region of interest applied on MRI image," in Proc IEEE 1st International Conference on TeleRadiology-Telemedicine, Bioinformatics and Biomedical Engineering, July 2007, pp. 1331-1334.

[13] S. Koliwad et al., "Lossless compression of digital mammography using fixed block segmentation and pixel grouping," in Proc. IEEE 6th Indian Conference on Computer Vision, Graphics \& Image Processing Bhubaneswar, December 2008, pp. 201-206.

[14] M. Y. Javed and M. Habib Khan, "Wavelet based medical image compression through prediction," in Proc. IEEE International Multi Topic Conference, Karachi, December 2008, pp. 155-159.

[15] P. Bharti, S. Gupta, and R. Bhatia, "Comparative analysis of image compression techniques a case study on medical images," in Proc IEEE International Conference on Advances in Recent Technologies in Communication and Computing, Kottayam, Kerala, November 2009, pp. 820-822.

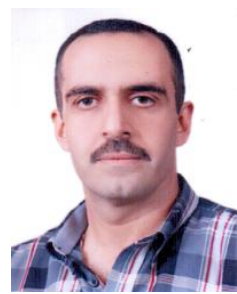

Arif Sameh Arif was born in Baghdad, Iraq, in 1970. He received his B.SC. degree in mathematics from the Al Mustansiria University, Baghdad in 1992. He received his M.SC. degree in computer science from University of Technology, Baghdad in 2004. He is currently a Ph.D. candidate at Multimedia University, Malaysia.

His research interests are in Image Processing, and medical Image compression.

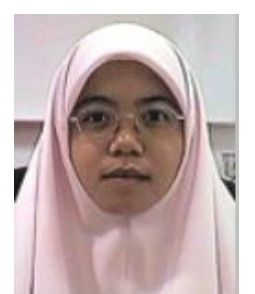

Sarina Mansor received her B. Eng. (Hons) electronic \& electrica from University College London in 1998, and M.Eng.Sc. degree from Multimedia University in 2004. She completed her DPhil in Engineering Science from University of Oxford, UK in 2009. Her research interests are Image Analysis, Wavelet Analysis, and Medical Image Analysis.

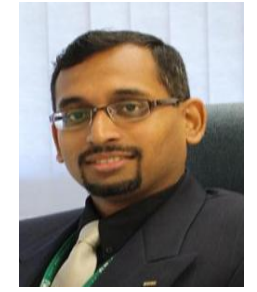

Rajasvaran Logeswaran received his $\mathrm{PhD}$. He is an associate professor and dean of engineering, science and technology at Nilai University, Malaysia and also an Adjunct staff at Multimedia University, Malaysia. Having received several scholarships and grants from Telekom Malaysia, Jaffnese Cooperative Society, Brain Gain Malaysia, Brain Korea 21 and the Ministry of Science, Technology and Innovation, Malaysia, Logeswaran successfully gained his undergraduate B.Eng. (Hons) Computing degree from the University of London, United Kingdom, postgraduate degrees from Multimedia University and conducted post-doctorate research at Soongsil University, Korea. His areas of interest include medical image analysis, data compression and neural networks.

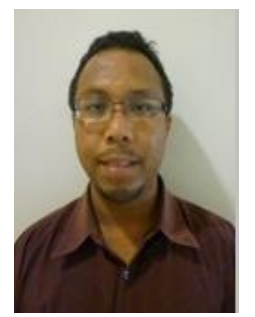

Hezerul Abdul Karim received B.Eng. degree in electronics with communications from University of Wales Swansea, UK, in 1998. He received his M. Eng Science degree from Multimedia University, Malaysia in 2003. He is a lecturer at Multimedia University since 2002 and now a Senior Lecturer since 2010. He obtained his $\mathrm{PhD}$ degree from Center for Communication Systems Research (CCSR), University of Surrey, UK in 2008. His research interests include telemetry, 2D/ $3 \mathrm{D}$ image/video processing and transmission, error resilience and multiple description video coding. 